\title{
Letters
}

Website: www.bmj.com

Email: letters@bmj.com

\section{Majority view of climate scientists is that global warming is indeed happening}

EDITOR-Gardner states that the scientific basis of climate change is uncertain and that there are major differences of opinion among climatologists about whether climate change is likely to occur and its potential magnitude. Inevitably, with an issue of such complexity there is bound to be scientific debate, but the Intergovernmental Panel on Climate Change, which we quoted in our articles, is a major international collaboration: it has involved the participation of over 2500 scientists from around the world. Many of the most vociferous sceptics have received funding from fossil fuel industries, which clearly have a vested interest in opposing changes in policy that might result in shifts away from fossil fuels. ${ }^{2}$

The evidence that Gardner quoted against climate change-that satellite data have shown $0.13^{\circ} \mathrm{C}$ global cooling between 1979 and 1994 and that the Arctic has been cooling-is mistaken. Satellite measurements are in good agreement with records of surface temperature for 1979-94. ${ }^{3}$ Once the transient effects of volcanoes and the El Niño-southern oscillation are removed, upward global trends of $0.09^{\circ} \mathrm{C}$ per decade from satellite data and $0.17^{\circ} \mathrm{C}$ per decade from surface data are obtained. ${ }^{4}$ This is reasonable agreement, especially when one realises that the satellite measurement is a weighted average over the atmosphere column from the surface to $7 \mathrm{~km}$ altitude and temperature changes in the midtroposphere may well differ from those at the surface. Furthermore, although temperatures have fallen in Greenland, they have risen by similar amounts over much of the remaining Arctic, notably in Siberia, northern Canada, and Alaska. ${ }^{5}$ There is compelling evidence that the average global temperature has increased by about $0.6^{\circ} \mathrm{C}$ since the industrial revolution. Indeed, nine of the 11 hottest years this century have occurred since 1985. More importantly, climate models suggest that substantial global warming $\left(1.0-3.5^{\circ} \mathrm{C}\right)$ will have occurred by the end of the next century with projected increases in use of fossil fuels. This underlines the need for a precautionary approach to limit emissions of greenhouse gases as well as increased investment in research on climate change and its potential impacts.

Finally, we must emphasise that the prospect of large scale climate and environ- mental changes necessitates the contingent assessment of future risks. Epidemiologists (like agricultural scientists, hydrologists, urban planners, and others) cannot reasonably dispute the majority view of climate scientists. Rather, they must base their risk assessment on the climate change scenarios projected by those experts.

Andrew Haines Professor of primary care Department of Primary Care and Population Sciences, Royal Free and University College London Schools of Medicine, London NW3 2PF

Anthony J McMichael Professor of epidemiology Sari Kovats Research fellow

Department of Epidemiology and Population Health, London School of Hygiene and Tropical Medicine, London WC1E 7HT

Mark Saunders Lecturer

Benfield Greig Hazard Research Centre, Department of Space and Climate Physics, University College London, Holmbury St Mary, Dorking, Surrey RH5 6NT

1 Gardner G. Many climate change scientists do not agree
that global warming is happening. BMJ 1998;316:1164 that global in

(11 April.)

3 Pearce F. Greenhouse wars. New Scientist 1997;155:38-43. Now York: Cambridge University Press, 1997:48.

New York: Cambridge University Press, 1997:48.
4 Jones PD. Recent warming in global temperature series. Jones PD. Recent warming in glo
Geophys Res Lett 1994;21:1149-52.

5 Intergovernmental Panel on Climate Change. Technical summary. In: Houghton JT, ed. Climate Change 1995: The science of climate change. New York, Cambridge University Press, 1996:27. (Second assessment report of the Intergovernmental Panel on Climate Change: contribution of working group I.)

\section{PACT data for dispensed drugs linked to NHS numbers are available now}

EDITOR-The Information in Practice article by Majeed et al provided important insights into what can and cannot be obtained from prescribing analysis and cost (PACT) data. ${ }^{1}$ Their conclusion that the addition of the NHS number to prescriptions would not be feasible until all general practices are fully computerised, however, is incorrect. Since 1990 the Medicines Monitoring Unit has been adding the NHS number (formerly the community health number in Scotland) to all prescriptions for the Tayside region of Scotland (400 000 people) and for prescriptions for selected drugs covering the whole of Scotland (5.5 million people). ${ }^{2}$ This has been achieved centrally at the unit by using purpose built software that enables the number to be accurately and rapidly ascribed on the basis of limited information on the patient's name and address and, optionally, the prescribing general practitioner's identifier. Such person specific data have been used in record linkage studies for the purpose of drug safety and pharmacoeconomic and outcomes research.

With funding from the Scottish Office, the Medicines Monitoring Unit has recently developed and tested an electronic system linking retail pharmacies to a central database of NHS numbers. ${ }^{3}$ This system enables pharmacists to draw down patients' NHS numbers rapidly, thus enabling the creation of highly accurate drug records for patients. Because about seven tenths of patients are loyal to one pharmacy, this system is inexpensive. It has been piloted in five pharmacies and has accurately added the NHS number to over 150000 prescriptions and correctly transmitted the data on dispensed drugs back to a central database with no increase in workload to the pharmacists. ${ }^{4}$ The addition of the NHS number at the time of dispensing is sensible as nearly all pharmacists are computerised and prescriptions written by hand on home visits, out of hours, by untrained locums, and when the practice computer fails are all captured. In addition, data on drugs dispensed are captured, which are often significantly different from data on drugs prescribed. ${ }^{5}$

The technology for capturing data on drugs dispensed that are specific to NHS numbers is available now. The NHS number

\section{Advice to authors}

We prefer to receive all responses electronically, sent either directly to our website or to the editorial office as email or on a disk. Processing your letter will be delayed unless it arrives in an electronic form.

We are now posting all direct submissions to our website within 72 hours of receipt and our intention is to post all other electronic submissions there as well. All responses will be eligible for publication in the paper journal.

Responses should be under 400 words and relate to articles published in the preceding month. They should include $\leqslant 5$ references, in the Vancouver style, including one to the BMJ article to which they relate. We welcome illustrations.

Please supply each author's current appointment and full address, and a phone or fax number or email address for the corresponding author. We ask authors to declare any conflicts of interest.

Letters will be edited and may be shortened.

\section{www.bmj.com}

letters@bmj.com 
can make a dramatic difference to patient care, and its use on all items of patient information should be encouraged. With the use of digital certificates (to ensure user identification and message authenticity), transaction logging (to provide an audit trail of user activity), and behavioural surveillance programs (to detect patterns of improper use), such a system need not pose a threat to confidentiality.

T M MacDonald Clinical reader

J Parkinson Client services director

P G Davey Clinical reader

A D Morris Senior lecturer

D G McDevitt Professor

M M McGilchrist Senior computer programmer Medicines Monitoring Unit (MEMO), Department of Clinical Pharmacology and Therapeutics, Ninewells Hospital and Medical School, Dundee DD1 9SY

1 Majeed A, Evans N, Head P. What can PACT tell us about prescribing in general practice? BMJ 1997;315:1515-9. (6 December.)

2 MacDonald TM, McDevitt DG. The Tayside Medicine Monitoring Unit. In: Strom BL, ed Pharmacoepidemiology 2nd ed. Chichester: John Wiley, 1994:245-55.

3 McGilchrist MM MacDonald TM Automatic capture of community prescribing. Pharmacoepidemiol Drug Safety 1997;6(suppl 2):S78. [Abstract 165.]

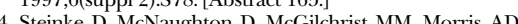
Davy PG, Mac Don D, McGilchist MM, Morris AD, Davey PG, MacDonald TM. Capturing person-specific dispensed prescribing data from community pharmacies assessing the change in workload by a time and motion study. Pharmacoepidemiol Drug Safety 1997;6(suppl 2):S80. Abstract 169.]

5 Beardon PHG, McGilchrist MM, McKendrick AD, McDevitt DG, MacDonald TM. Primary non-compliance with prescribed medications in primary care. BMJ 1993; 307:846-8

\section{There is no such thing as ageing}

\section{Ageing has been defined as to grow or make old}

EDITOR-In an effort to delay my own ageing process, I have struggled to understand Peto and Doll's argument that there is no such thing as ageing. Their case seems to be predicated on their second paragraph, where they assert: "What the major diseases of adult life have shared for tens of millions of years is a common set of evolutionary pressures tending to relegate them to old age... . Natural selection acts much more strongly against death in early adult life than against death in old age."

Apart from this being a circular argument, which in its conclusion is more of a description than an explanation, it fails to account for some facts. Thus, if the underlying mechanism is an evolutionary one acting over such a long time span, how are we to understand the dramatic changes in life expectancy that have occurred in industrial societies-even within the living memories of your older readers? Butler's article in the same issue shows this clearly. ${ }^{2}$ Similarly, Peto and Doll's case rests uneasily with that of Grimley Evans, who describes hale elderly men in biblical and Greek history. ${ }^{3}$ Perhaps those who survived to be octogenarians then were fitter than those today. Most would settle for Moses's epitaph: he "was an hundred and twenty years old when he died: his eye was not dim, nor his natural force abated." ${ }^{4}$ These changes in life expectancy (possibly in both directions) are almost contemporary in an evolutionary time scale. They certainly can have had nothing to do with a postulated teleological pressure.

Peto and Doll also engage in some diverting mathematical juggling between mice and men. While they admit that for their thesis the difference between a billion and a trillion does not matter, their extrapolations are likely to be equally immaterial.

It is an old (you might say aged) trick to take a common, generally understood term and so hedge your definition of it with qualifications that it is robbed of its meaning. I would settle for the definition of age and ageing in the Shorter Oxford Dictionary of 1933-"to grow or make old."

D E B Powell Retired consultant pathologist Ogmore by Sea, Bridgend CF32 OPT

Conflict of interest: I am aged.

1 Peto R, Doll R. There is no such thing as aging. BMJ 1997 ; 315:1030-2. (25 October.)

Butler RN. Population aging and health. BMJ 1997; 315:1082-5. (25 October.)

3 Evans JG. Geriatric medicine: a brief history. BMJ 1997; 315:1075-7. (25 October)

4 The Holy Bible. Deuteronomy 34:7.

\section{Authors' reply}

EDitor-Over several decades the human body undergoes various changes, such as greying of hair, baldness, accumulation of somatic mutations in particular cells, menopause, and so on. Our claim is that there is no good reason to believe that all these age related changes arise from a single common mechanism that could usefully be termed ageing-hence the title of our editorial. Turning from the individual to the species, any particular condition that is likely to cause death in the years of parenthood will tend to get pushed to older ages, but the mechanisms by which evolutionary pressures push diseases into old age may be very different for different conditions. Again there is no good reason to believe that this evolutionary delay of the usual age of onset of disease entails just a single process.

Evolutionary changes that substantially affect the lifespan may well take millions of years. In the few thousand years since written records began there can have been little change in the main genetic determinants of the human lifespan, so we still cannot hope for much more than the biblical three score years and ten. But, what has changed greatly during historical times, with particularly favourable changes in recent decades, is the extent to which we are exposed to avoidable causes of death in youth or middle age. And to avoid any conflict of interest or otherwise with Powell, we here adopt the definition of middle age in Chambers English Dictionary as the period "between youth and old age, variously reckoned to suit the reckoner."

Richard Doll Emeritus professor of medicine Richard Peto Professor of medical statistics Clinical Trial Service Unit, Nuffield Department of Clinical Medicine, Radcliffe Infirmary, Oxford OX2 6HE

\section{Can students learn comparable clinical skills in general practice and hospital settings?}

Students are discriminating consumers of educational experience

EDITOR-Murray et al used a randomised crossover trial to study the acquisition of clinical skills in general practice. ${ }^{1}$ Our experience with the use of randomised trials in medical education has shown that they may be unacceptable to students. In 1996, teaching by general practitioners was introduced into the ear, nose, and throat curriculum of the Imperial College School of Medicine at St Mary's. The aim of such teaching was to give students a grounding in the basic skills of taking a history and examining the ear, nose, and throat. All the general practitioners were trained in teaching skills, and they attended a refresher course in ear, nose, and throat medicine. The students were allocated to the general practitioners for three sessions during the four week course.

A study was designed to ascertain the most effective form of teaching. After a pilot phase in which all the students were taught by the general practitioners (to ensure that the tutors were experienced in teaching) the students were randomised to either teaching by the general practitioners or the old curriculum. An objective structured clinical examination was planned at the end of every two rotations. After the first rotation the students refused to be randomised to the old curriculum. They explained that unless they were taught by a general practitioner they had no systematic training in ear, nose, and throat examination. They refused to participate and the trial was abandoned. All students now receive training by general practitioners, which remains extremely popular.

This experience shows that medical students are discriminating consumers of educational experience and will seek out teaching that meets their learning needs. A crossover trial, which ensures that students have the same experience, is difficult to construct in a specialty allocated a short time. Depriving students of an experience available to their peers is difficult to justify ethically and, in our trial, students attended sessions by the general practitioners to which they had not been allocated. Finally, any pilot phase that includes teaching will cause a compensatory adjustment in the curriculum.

Unless medical students can be offered equivalent educational experience within the structure of a trial they are unlikely to feel that it is in their best interests to cooperate fully. Researchers should consider how their ethical obligations to students can be met while continuing high quality research in education.

Sarah Hartley Clinical lecturer in general practice Anita Berlin Senior lecturer in general practice Neil Tolley Consultant ear, nose, and throat surgeon Imperial College School of Medicine at St Mary's, London W2 1PF

s.hartley@ic.ac.uk 
1 Murray E, Jolly B, Modell M. Can students learn clinical method in general practice? A randomised crossover trial based on objective structured clinical examinations. $B M$ J 1997;315:920-3. (11 October.)

\section{Availability of resources may have been a key factor}

EDITOR-The paper by Murray et al on learning clinical method in general practice showed that anything can be done when enough resources are available. ${ }^{1}$ Certainly the clinical skills acquired by students based in a hospital were not greatly different from those acquired by students based in the community, but the resources required for this result were widely disparate: firstly, students were generally present in the general practices for half a year; secondly, the tutor posts were filled only by those enthusiastic and dedicated enough to provide protected learning time and undergo training in teaching methods; thirdly, one to one teaching was provided by dedicated tutors; and, fourthly, no junior staff were involved. In other words, the general practitioner cohort was taught by a highly motivated group of people and the hospital cohort was taught by hospital staff for whom teaching was an accepted (albeit not always preferred) part of the job.

Others have suggested that teaching clinical students in the community is more expensive than teaching them in a hospital setting. ${ }^{2}$ If general practitioners are paid specifically to provide protected teaching time this seems perfectly natural. ${ }^{1}$

I am not denying that there is a place for the teaching of knowledge, skills, and attitude in the community, but I believe that this study compares an enthusiastic and well resourced group with a sometimes reluctant and perhaps underfunded group. If these differences were reconciled would clinical students taught in hospital excel?

Jonathan Hobson Fifth year clinical student Keble College, Oxford OX1 3PG jonathan.hobson@keble.oxford.ac.uk

1 Murray E, Jolly B, Modell M. Can studemts learn clinical method in general practice? A randomised crossover tria based on objective structured clinical examinations. $B M J$ 1997;315:920-3. (11 October.)

2 Murray E, Jinks V, Modell M. Community-based education feasibility and cost. Med Educ 1995;29:66-71.

\section{Like should be compared with like in a randomised crossover trial}

EDITOR-We are concerned about the conclusions drawn in the article by Murray et $\mathrm{al}^{1}$ as it is imperative that researchers make sure they are comparing like with like in a randomised crossover trial.

The study purported to compare the clinical skills teaching of first year medical students in general practice with that in a hospital environment, and it concluded that students can learn just as effectively in a general practice setting as they can in a hospital setting. Although the results show this to be the case, the paragraph on the study in This Week in the $B M J$ seems to give a liberal interpretation in saying that [the results show] students acquire clinical skills at least as well in general practice. The implication is clear that the authors thought that being taught by a general practitioner was better.

These statements, however, are wrong as the study did not compare similar teaching in the two settings. Firstly, the students allocated to be taught in general practice were always taught by one person and not by any of a number of tutors as is the case in the hospital setting. Secondly, the hospital tutors could have been a member of the consultant staff, senior academic staff, or junior staff whereas in the general practice setting the implication is that general practitioner registrars were not involved. Finally, and most importantly, the general practitioner tutors were paid for specific sessions for teaching, which was in protected time. Because hospital doctors' contracts include teaching they would be fortunate to have protected teaching time available, and many would find the increasing burden of service commitments intruding on their teaching slots.

It would be surprising if those being paid to teach in protected time were less enthusiastic than those being asked to include increasing amounts of teaching in an already crowded timetable. As the odds in this study seem to be in favour of general practice teaching it is amazing that it did not do better than it did.

David M Reid Reader in rheumatology

Medical School Buildings, University of Aberdeen, Aberdeen AB25 2ZD

1 Murray E, Jolly B, Modell M. Can students learn clinical method in general practice? A randomised crossover tria based on objective structured clinical examinations. $B M$ 1997;315:920-3. (11 October.)

\section{Authors' reply}

EDITOR-We agree with Hartley et al that there can be ethical problems with randomising students while evaluating innovations in teaching. As with trials of new treatments, it is ethical when there is genuine uncertainty over which treatment (teaching programme) is better, or when the aims of each programme are the same. We used a randomised crossover approach partly to avoid ethical problems and partly to ensure a balanced design.

In education, randomised crossover trials do not have the same attributes as those used in medical research, and it can be difficult to use them appropriately or to draw precise comparisons. ${ }^{1}$ Hobson and Reid both argue that we were not comparing like with like. This is true, and we went to considerable lengths to describe the teaching in both locations, highlighting the similarities and differences. The pattern of hospital work is different from that in a community practice. This forestalls the construction of largely identical treatments merely for the purposes of an experiment. Both programmes, however, were representative of teaching in their respective environments, and both had identical objectives.

One original purpose of this study was to address widespread scepticism over whether students could achieve these objectives in general practice. Hospital trusts do receive resources to offset teaching costs (the service increment for teaching is currently about $£ 50000$ for each clinical student per annum). Unlike teaching hospitals, which receive funding for both facilities and placements, at present general practitioners receive funding for placements only (currently one fifth of the service increment for teaching). In 1996-7 the Anglian region, in which Hobson will receive his clinical training, received almost $£ 5$ million for clinical placements and £21 million for facilities. In general practice, teaching can usually only be done by a general practitioner. In hospital the wider range of teachers, including junior doctors, could be seen as an opportunity to provide more contact hours for students. We agree that protected teaching time is important to the success of all teaching; this is why we targeted resources to provide it in general practice. We strongly support moves by hospital teachers to do the same.

Our view is that general practice and hospital teaching are complementary. Our subsequent work suggests that some objectives of the first clinical year are better achieved in hospital and others in the community. More educational research is certainly needed to clarify the relative strengths and weaknesses of innovative and traditional teaching to allow rational planning of the curriculum. Tomorrow's doctors deserve the best medical education possible.

Elizabeth Murray Senior lecturer in primary health care

Michael Modell Professor of primary health care Department of Primary Care and Population Sciences, Whittington Hospital, London N19 5NF

Brian Jolly Director

Medical Educational Unit, University of Leeds LS2 9NL

Senn S. Cross-over trials in clinical research. Chichester: Wiley, 1993.

\section{Compression ultrasonography for diagnosing deep vein thrombosis}

\section{One examination of whole leg is better than two of selected parts}

Editor-We advise caution in adopting the proposed abbreviated examination protocol for detecting deep vein thrombosis in the leg. ${ }^{12}$ Our experience using duplex ultrasonography to study the natural course of deep vein thrombosis leads us to make the following points.

Firstly, it is incorrect that calf deep vein thrombosis cannot be accurately diagnosed by duplex ultrasonography. Many reports from expert centres refute such an opinion, although we agree that the skill and experience of the sonographer is important.

Secondly, about $14 \%$ of isolated calf deep vein thromboses propagate into the proximal veins. Others have reported an incidence of up to $20 \%$ propagation. $^{3}$ 
Thirdly, because of the varying attitudes to management of isolated calf deep vein thrombosis some will argue against the need to investigate these veins on the basis that pharmacological treatment is not contemplated.

Fourthly, isolated superficial femoral deep vein thrombosis without involvement of the popliteal (distal) or common femoral (proximal) venous segments can occur. This presentation may cause few clinical signs and symptoms, and the thrombus only partially occludes the vein with poor adhesion of the clot to the vein wall. The frequency of this presentation makes it mandatory to examine the whole length of the veins in the thigh. The additional time required is minimal, but the information obtained is important.

Fifthly, the editorial scenario of office based worldwide facilities to diagnose deep vein thrombosis accurately ${ }^{2}$ requires sufficient technical skill and experience with audit control to assure standards at each of these offices. Reports of therapeutic results could otherwise be confusing.

The examination protocol recommended by Cogo et al has the attraction of saving time but does so at the expense of ignoring $15-25 \mathrm{~cm}$ of vein in the thigh (which might take 2 minutes to examine) and excluding the calf veins, which have a potential to propagate thrombi in $14-20 \%$ of cases. Re-investigation after a week detected a further $1 \%$ of deep vein thromboses in those venous segments being examined. We believe that one full examination of the veins of the leg would provide more comprehensive information, take less time, and be more cost effective than investing in two examinations over a week evaluating only short selected segments of the venous system.

D E FitzGerald Consultant physician

A M O'Shaughnessy Vascular technologist

Vascular Medicine Unit, James Connolly Memorial Hospital, Dublin 15, Ireland

1 Cogo A, Lensing AWA, Koopman MMW, Piovella F, Siragusa S, Wells PS, et al. Compression ultrasonography for diagnostic management of patients with clinically suspected deep vein thrombosis: prospective cohort study. BMJ 1998;316:17-20. (3 January.)

2 Davidson BL, Deppert EJ. Ultrasound for the diagnosis of deep vein thrombosis: where to now? BMJ 1998;316:2-3 (3 January.)

3 O'Shaughnessy AM, FitzGerald DE. The value of duplex ultrasound in the follow-up of acute calf vein thrombosis Int Angiology 1997;16:1421-46.

\section{Other tests should not be abandoned}

Editor-Cogo et al describe a technique which apparently reduces the need for conventional venography in the investigation of suspected deep vein thrombosis. ${ }^{1}$

We recently reported that duplication of the superficial femoral vein or more complex anomalies are found in $46 \%$ of patients having venography in our hospital after normal results on Doppler ultrasonography of the thigh. ${ }^{2}$ Although Cogo et al argue that "assessment of the proximal veins along their entire course in the thigh results in loss of specificity and is not indicated because symptomatic patients with venous thrombosis usually have large thrombi affecting at least the popliteal or common femoral vein," ${ }^{3}$ our study showed that duplicated superficial femoral veins are a common and important exception to this view. ${ }^{2}$ Furthermore, these complex superficial venous systems result in false negative colour flow Doppler ultrasound results in $6 \%$ of cases compared with $2 \%$ of cases with a single superficial femoral vein.

In addition, Cogo et al use only clinical symptoms as end points of pulmonary embolism and long term deep vein thrombosis. The incidence of subclinical pulmonary embolism is not known in their patient group. Silent pulmonary embolic disease may, however, have long term effects on morbidity, including pulmonary hypertension. Finally, there is the question of the postphlebitic syndrome, which occurs not only in patients with distal popliteal thrombus but in those with isolated calf vein thrombosis. Cogo et al and the accompanying editorial by Davidson and Deppert ${ }^{4}$ do not consider this to be an end point, but the incidence of imperforate deep penetrating calf veins and the postthrombotic syndrome after isolated calf thrombosis is considered by some centres as sufficient indication for anticoagulation, while others recommend graduated compression stockings. ${ }^{5}$ This is obviously not an issue in centres that consider that ultrasonography is sufficiently sensitive to exclude isolated calf thrombi.

Many unanswered questions remain. We do not know the real importance and natural course of isolated calf thrombus. The complex anatomy of the superficial femoral venous system is poorly appreciated. New non-imaging diagnostic strategies (such as dimers) are in their infancy. Although the results of Cogo et al's study are encouraging, it may be premature to support a single, rather limited, ultrasound examination.

Jonathan $\mathbf{H}$ Gillard Clinical lecturer

Nicholas J Screaton Specialist registrar

Laurence H Berman Senior lecturer

Department of Radiology, School of Clinical

Medicine, University of Cambridge, Addenbrooke's Hospital, Cambridge CB2 2QQ

1 Cogo A, Lensing AWA, Koopman MMW, Piovella Siragusa S, Wells PS, et al. Compression ultrasonography

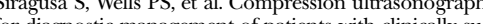
for diagnostic ma pected deep vein thrombosis. 20. (3 January.)

2 Screaton NJ, Gillard JH, Berman LH, Kemp PM. Complex superficial veins: a source of error in the investigation of venous thrombosis. Radiology 1998;206:397-401.

3 Cogo A, Lensing AWA, Prandoni P, Hirsch J. Distribution of thrombosis in patients with symptomatic deep vein thrombosis: implication for simplifying the diagnostic process of compression ultrasound. Arch Int Med 1993;153:2777-80.

4 Davidson BL, Deppert EJ. Ultrasound for the diagnosis of deep vein thrombosis: where to now? BMJ 1998;316:2-3. (3 January.)

5 Wood AJJ. Management of venous thromboembolism. N Engl J Med 1996;335:1816-28.

\section{Venography is more accurate}

EDITOR-Cogo et al's study may further exacerbate potentially dangerous misconceptions about the best method of diagnosing deep venous thrombosis. ${ }^{1}$ Some of the issues require clarification.

Firstly, the reported incidence of subsequent major thromboembolic events is a crude method of assessing the accuracy of ultrasonography. In the study design the authors promise confirmatory venography to assess the positive predictive value of ultrasonography, but this is not reported later in the paper. A paper last year by one of the authors indicated a significant false positive rate for compression ultrasonography with a positive predictive value of only $71 \%{ }^{2}$

Secondly, although this study's results seem to improve on previous non-invasive efforts, the author's concede that intrinsic problems with ultrasonography necessitate a second scan. However, scant attention is paid to the implications of this strategy. In most UK centres a single scan is all that is on offer $^{3}$ and a negative result is taken to equate with absence of deep vein thrombosis. Thus the inevitable watering down of Cogo et al's protocol may have serious clinical and medicolegal consequences.

Thirdly, Cogo and colleagues may unwittingly add to the chaos about the choice of further investigations for patients with potential pulmonary embolism who have indeterminate lung scans and no limb symptoms. The recent guideline from the British Thoracic Society which advocates the use of ultrasound for these patients exemplifies this confusion. ${ }^{4}$ The recommendation is made despite several studies having shown that ultrasonography has an appalling sensitivity compared with contrast venography in high risk patients with no limb symptoms. In Lensing et al's study sensitivity was as low as $47 \%{ }^{2}$

The authors correctly state that contrast venography is the standard by which all other tests are measured but go on to dismiss it as invasive while invoking the bogey man of contrast reaction. Venography is as invasive as taking a blood sample, and reactions to modern contrast materials are as rare as hen's teeth. Slavish pursuit of noninvasive alternatives to an accurate test which can never be considered a serious intervention may not be worth the trade off.

John $\mathbf{H}$ Reid Consultant radiologist

David J Hardwick Consultant radiologist Borders General Hospital NHS Trust, Melrose, Roxburghshire TD6 9BS

1 Cogo A, Lensing AWA, Koopman MMW, Piovella F, Siragusa S, Wells PS, et al. Compression ultrasonography for diagnostic management of patients with clinically suspected deep vein thrombosis: prospective cohort study. BMJ 1998;316:17-20. (3 January)

2 Lensing AWA, Doris CI, McGrath FP, Cogo A, Sabine NJ Ginsberg J, et al. A comparison of compression ultrasound with color Doppler ultrasound for the diagnosis of symptomless postoperative deep vein thrombosis. Arch Intern Med 1997;157:765-8.

3 Burn PR, Blunt DM, Sanson HE, Phelan S. The radiological investigation of suspected lower limb deep vein thrombosis. Clin Radiol 1997;52:625-8.

4 Corris P, Ellis D, Foley N, Miller A for British Thoracic Society, Standards of Care Committee. Suspected acute pulmonary embolism: a practical approach. 1997;52(suppl 4)

\section{Colour Doppler provides useful information}

EDITOR-Cogo et al's protocol using compression ultrasonography at just three sites to diagnose deep vein thrombosis requires some comment.

Firstly, the authors suggest that although only $3 \%$ of patients with deep vein thrombosis were identified by the repeat scan at one week, a repeat scan after all normal results is justified by the potential for fatal pulmonary embolism. Vaccaro et al used the same 
simplified compression ultrasound technique and detected a deep vein thrombosis at presentation in 376 of 1355 symptomatic patients. ${ }^{2}$ They, however, repeated the compression ultrasound test at 72 hours in only those patients with persistent clinical signs and a negative initial test result. Deep vein thrombosis was found in nine of these 86 patients. Follow up of all patients with initial negative ultrasound results over 8-33 months showed that only five patients $(0.48 \%)$ suffered thromboembolic complications (compared with $0.9 \%$ reported by Cogo et al). Limiting repeat scans to patients still having clinical signs therefore seems appropriate and would avoid a large unnecessary workload.

Secondly, colour Doppler ultrasonography is now the first line investigation for diagnosing deep vein thrombosis. ${ }^{3}$ A survey of UK radiology departments found that 51 of 111 departments $(46 \%)$ use colour Doppler ultrasound $90 \%$ or more of the time and 66 departments $(59 \%)$ used it for $50 \%$ of cases. ${ }^{4}$ Baxter showed that colour Doppler allows the classification of thrombi into occlusive and non-occlusive types, which may be of prognostic significance. ${ }^{3}$ A recent study using colour Doppler showed similar sensitivity and specificity for both above and below knee deep vein thromboses. ${ }^{5}$ Colour Doppler should reduce the number of false positive results and reduce the need for a repeat test if the clinician anticoagulates deep vein thromboses in the calf. We believe compression ultrasonography for the femoral and popliteal veins plus colour Doppler of the thigh and calf should be the first imaging investigations in the diagnosis of lower limb deep vein thrombosis.

Stephen Wolstenhulme Senior radiographer

Simon Richards Vascular somographer

Michael Weston Consultant radiologist

Ultrasound Department, St James's University

Hospital, Leeds, LS9 7TF

1 Cogo A, Lensing AWA, Koopman MMW, Piovella F Siragusa S, Wells PS, et al. Compression ultrasonography for diagnostic management of patients with clinically suspected deep vein thrombosis: prospective cohort study pected deep vein thrombosis: $\mathrm{p}$

2 Vaccaro JP, Cronan IJ, Dorfman GS. Outcome analysis of patients with normal compression ultrasound examinapatients with normal compress
tions. Radiology 1990;175:645-9.

3 Baxter GM. The role of ultrasound in deep venous thromBaxter GM. The role of ultraso
bosis. Clin Radiol 1997;52:1-3.

bosis. Clin Radiol 1997;52:1-3.
4 Burn PR, Blunt DM, Sanson HE, Phelan MS. The Burn PR, Blunt DM, Sanson HE, Phelan MS. The
radiological investigation of suspected lower limb deep venous thrombosis. Clin Radiol 1997;52:625-8.

5 Bradley MJ, Spencer PA, Alexander L, Milner GR. Colour flow mapping in the diagnosis of the calf deep vein thrombosis. Clin Radiol 1993;47:399-402.

\section{Protocol is safe}

EDITOR-We agree with Cogo et al that it is safe to withhold anticoagulant treatment from patients with suspected deep vein thrombosis who have a normal ultrasound scan result. ${ }^{1}$ We have used ultrasound scanning as our primary investigation for suspected deep vein thrombosis since 1995 . To confirm that we were not missing significant thromboses by adopting this practice we performed a clinical audit.

The audit was designed to identify all patients who died or were readmitted within three months of a negative ultrasound result in a district general hospital. Over 12
Patients who were readmitted or died within three months of a negative ultrasound result

Time from

negative scan
to readmission

(years) or death (days)

\begin{tabular}{ll}
\hline Reason for admission & \\
\hline Suspected right calf deep vein & 52
\end{tabular}

\begin{tabular}{llr}
\multicolumn{1}{c}{ thrombosis $^{\star}$} & 37 & 5 \\
\hline 2 In labour & 82 & 14 \\
\hline 3 Cellulitis* & 67 & 14 \\
\hline 4 Rectal bleeding & 75 & 18 \\
\hline $\begin{array}{l}\text { B Bilateral common iliac artery } \\
\text { angioplasties }\end{array}$ & &
\end{tabular}

angioplasties

\begin{tabular}{lll}
\hline 6 In labour & 27 & 18 \\
\hline 7 Prostate cancer & 83 & 22 \\
\hline 8 Chest pain & 76 & 24 \\
\hline 9 Parkinson's disease & 76 & 24 \\
\hline 10 Atypical angina & 71 & 30 \\
\hline 11 Fall & 82 & 33 \\
\hline 12 In labour & 31 & 42 \\
\hline 13 Collapse & 68 & 47 \\
\hline 14 Metastatic squamous cell & 76 & 58
\end{tabular}

14 Metastatic

carcinoma

\begin{tabular}{lll}
\hline 15 Metastatic disease & 76 & 62
\end{tabular}

\begin{tabular}{lll}
\hline 16 Pneumonia & 74 & 69 \\
\hline 17 Back pain & 81 & 70
\end{tabular}

\begin{tabular}{lll}
\hline 17 Back pain & 81 & 70 \\
\hline 18 Fractured wrist & 80 & 72 \\
\hline 19 Asthma, shortness of breath & 50 & 86
\end{tabular}

19 Asthma, shortness of breath $\quad 50 \quad 86$

Cause of death

\begin{tabular}{lcc}
\hline 20 Bronchopneumonia & 80 & 4 \\
\hline 21 Multiorgan failure & 76 & 7 \\
\hline 22 Methicillin resistant & 78 & 16
\end{tabular}

Staphylococcus aureus endocarditis

23 Septicaemia, peritonitis, bowel $82 \quad 17$

perforation

24 Metastatic carcinoma of the vulva $91 \quad 62$

25 Bronchiectasis, pulmonary

fibrosis, systemic sclerosis

\begin{tabular}{lll}
\hline 26 Metastatic squamous cell & 68 & 64
\end{tabular}

carcinoma

\begin{tabular}{lll}
\hline 27 Metastatic squamous cell & 76 & 84
\end{tabular}

carcinoma of lung

28 Metastatic disease of breast $\quad 76 \quad 88$

*Repeat ultrasound scan on readmission. All gave negative results.

months a total of 424 patients had ultrasound scanning for a suspected deep vein thrombosis; 202 patients with negative results were randomly selected for the audit. Of these, 14 were excluded as they were already receiving anticoagulant treatment (three long term warfarin, 11 prophylactic heparin). Nineteen patients required readmission within three months of a negative result (table). In three a venous thrombosis was suspected on readmission, but in all cases a repeat ultrasound scan gave negative results. In all other cases the clinical history did not suggest a diagnosis of deep vein thrombosis or pulmonary embolus. Nine patients died within three months (confirmed by the registrar of births, deaths, and marriages, the local health authority, or the local family health services authority). One patient died within four days from bronchopneumonia. Postmortem examination found no evidence of venous thrombosis or pulmonary embolus. There were no cases of unexplained sudden death.

We were aware that using ultrasound alone we would miss venous thromboses below the bifurcation of the popliteal vessel. Although previous studies have shown that deep vein thrombosis below the knee can give rise to silent pulmonary emboli, ${ }^{2}$ serious complications of this type of thrombosis are rare. $^{3}$

We agree with Cogo et al that, with modern sensitive techniques, a negative ultrasound result is sufficient to exclude clinically significant venous thrombosis of the lower limb and that venography is unnecessary. We would, however, question the role of a repeat scan at seven days. In our series a second scan was carried out only if patients represented with persistent symptoms. We propose that routine scanning at seven days is unnecessary but agree that a larger study is required to confirm this.

Paul L Harper Consultant haematologist

Lorna Watson Clinical audit officer

Roy Bannon Consultant radiologist.

West Suffolk Hospital, Bury St Edmunds IP33 2QZ

1 Cogo A, Lensing AWA, Koopman MMW, Piovella F Siragusa S, Wells PS, et al. Compression ultrasonography for diagnostic management of patients with clinically suspected deep vein thrombosis: prospective cohort study BMJ 1998;316:17-20. (3 January.)

2 Huisman MV, Buller HR, ten Cate JW, van Royen EA, Vreeken J, Kersten M, et al. Unexpected high prevalence of silent pulmonary embolism in patients with deep vein thrombosis. Chest 1989;95:498-502.

3 Philbrick JT, Becker DM. Calf deep vein thrombosis: a wolf in sheep's clothing. Arch Intern Med 1988;148:2131-8.

\section{Repeat testing is unjustified}

EDITOR-We agree with Cogo et al that their diagnostic strategy for deep vein thrombosis is safe. ${ }^{1}$ However, the strategy required repeat compression ultrasonography after one week when the initial test gave normal results. This safety measure was added to detect thrombi initially confined to calf veins, which would be missed by the first examination but would become detectable if they extended to the popliteal vein.

Overall, deep vein thrombosis was diagnosed in 412 patients (24\%). However, in all but 12 patients the diagnosis was made at the first examination. Thus, at the second testing 1278 of 1290 patients who had normal initial ultrasonographic findings had a normal result again, and the yield rate was just $0.9 \%$. Nevertheless, both the authors and Davidson and Deppert in the accompanying editorial ${ }^{2}$ judge that it is worth submitting all these patients to a second ultrasound scan despite the highly unfavourable ratio of 108 normal result against one abnormal result.

We feel that this view is wrong, especially because the value of an abnormal ultrasonographic finding in a population with such a low prevalence of deep vein thrombosis $(1 \%)$ is far from optimal. In this situation, assuming a specificity of venous compression ultrasonography of $97 \%,{ }^{3}$ the positive predictive value of an abnormal result will be as low as $25 \%$. Even if the specificity is $98 \%$ or $99 \%$, the predictive value will increase only to $33 \%$ or $50 \%$, respectively. Thus, among the 12 patients who were detected at the second investigation, at least half are likely to have been given anticoagulant therapy on the basis of a false positive result. 
In fact, few patients who were missed at the first examination could be added to the nine who experienced thromboembolic recurrence during the six month follow up. This would result in a cumulative six month thromboembolic risk of $1.6 \%$ (95\% confidence interval $1.0 \%$ to $2.6 \%$ ) for a simplified strategy with only one examination, an acceptable figure that could probably have been further improved by combining modern diagnostic tools such as prior clinical probability of deep vein thrombosis ${ }^{4}$ or plasma measurement of $\mathrm{D}$ dimer $^{5}$ with venous compression ultrasonography.

Henri Bounameaux Professor of medicine Arnaud Perrier Consultant physician

Division of Angiology and Haemostasis, Department of Internal Medicine, University Hospital of Geneva, Geneva, Switzerland

1 Cogo A, Lensing AWA, Koopman MMW, Piovella F, Siragusa S, Wells PS, et al. Compression ultrasonography for diagnostic management of patients with clinically suspected deep vein thrombosis: prospective cohort study. BMJ 1998:316:17-20. (3 January)

2 Davidson BL, Deppert FJ. Ultrasound for the diagnosis of deep vein thrombosis: where to now? BMJ 1998;316:2-3. (3 January.)

3 White RH, McGahan JP, Daschbach MM, Hartling RP. Diagnosis of deep-vein thrombosis using duplex ultraDiagnosis of deep-vein thrombosis using
sound. Ann Intern Med 1989;111:297-304.

4 Wells PS, Anderson DR, Bormanis J, Guy F, Mitchell M, Gray L, et al. Value of assessment of pretest probability of deep-vein thrombosis in clinical management. Lance 1997;350:1795-8.

5 Bounameaux H, de Moerloose P, Perrier A, Miron MJ D-dimer testing in suspected venous thromboembolism: an update. QJMed 1997;90:437-42.

\section{Authors' reply}

EDITOR-Objective diagnosis for patients with suspected venous thrombosis is indicated since a large proportion of these patients do not have this condition. Conventional ultrasonography is the non-invasive method of choice because of its wide availability, simplicity, and reproducible highly accurate diagnostic criterion (absence or presence of vein compressibility) for proximal vein thrombosis. ${ }^{1}$ However, ultrasonography cannot reliably detect the small proportion of patients with isolated calf clots. Undetected thrombi can remain active, enlarge, extend proximally, and if not detected by follow up tests cause pulmonary embolism. In the initial studies up to five tests were performed if the baseline test result was normal. ${ }^{1}$ Nowadays, we use only a single repeat ultrasound scan if the baseline result is normal. What are the possibilities to improve diagnostic management further?

Firstly, do more advanced duplex and colour ultrasound techniques detect isolated calf vein thromboses? Both techniques produce fascinating images, but interpretation of directional Doppler and colour flow mapping is subjective since they are not standardised or validated for venous disease. In fact, both techniques rely primarily on assessing vein compressibility. There is no sound evidence that these tests have an improved sensitivity for isolated calf thrombosis. No follow up studies have been done with specific duplex or colour flow criteria.

Secondly, is it safe to withhold anticoagulant treatment from patients with suspected venous thrombosis and a single normal ultrasound result? Vaccaro et al reported a retrospective analysis of this approach in symptomatic patients. ${ }^{2}$ No clearly defined protocol was used and active follow up was not performed. Their conclusion that venous thromboembolic complications occurred in less than $1 \%$ may be valid but could be the result of the "tip of the iceberg" phenomenon. It cannot be taken as justification for using a single ultrasound test.

Thirdly, can the single repeat ultrasound approach be further improved? The answer seems to be "yes." Wells et al showed that using a validated clinical decision rule as an adjunct to a normal baseline ultrasound result identified $55 \%$ of patients with a low likelihood of venous thrombosis. ${ }^{3}$ No further testing or treatment was done and follow up was uneventful. Additional testing identified venous thrombosis in an important proportion of the remaining patients. An alternative strategy involves assessment of $\mathrm{D}$ dimer (a fragment specific for the degradation of fibrin) after a normal baseline ultrasound result. In a large study normal ultrasound and $\mathrm{D}$ dimer results were found in over $85 \%$ of patients. No further testing was performed, no treatment was initiated, and long term follow up was uneventful (unpublished results). The small group of patients who had abnormal $\mathrm{D}$ dimer results were considered at risk for venous thrombosis; repeat ultrasonography at one week showed proximal vein thrombosis in $6 \%$ of them.

Anthonie W A Lensing Senior researcher Centre for Vascular Medicine, Academic Medical Centre, 1105 AZ Amsterdam, Netherlands.

Paolo Prandoni Senior researcher Istituto di Semeiotica Medica, University of Padua, 35128 Padua, Italy

1 Lensing AWA, Hirsh J, Buller HR Diagnosis of venous thrombosis. In: Colman RW, Hirsh J, Marder VJ, Salzman thrombosis. Tn. Colman RW, Hirsh J, Marder VJ, Salzman EW, eds. Hemostasis and ihombosis. basic principles and clinical practice. 3rd ed. Philadelphia.JB Lippineot, 1993:1297-32 2 Vaccaro JP, Cronan JJ, Dorfman GS. Outcome analysis of patients with normal compression US examinations. Radiology 1990;175:645-9.

3 Wells PS, Anderson DR, Bormanis J, Guy F, Mitchell M, Gray L, et al. Value of assessment of pretest probability of deep-vein thrombosis in clinical management. Lance 1997;350:1795-8.

\section{Attitudes to questioning in clinical practice may be changed}

EDITOR-Barrie and Ward suggest that a major barrier to the promotion of evidence based health care is the generation of questions by the doctor. ${ }^{2}$ The figure of only 2.4 questions asked per 10 patients should not astonish those who received a conventional medical education taught by authoritarian seniors in an atmosphere where questioning is not fostered.

While running a course on evidence based child health at Leeds University we collected data that suggest that questioning behaviour may be changed. Some weeks before the course, and again at the end, the participants (community paediatricians and paediatricians in training) completed a statistical competence questionnaire. Two supplementary questions were included: "What percentage of your clinical work do you
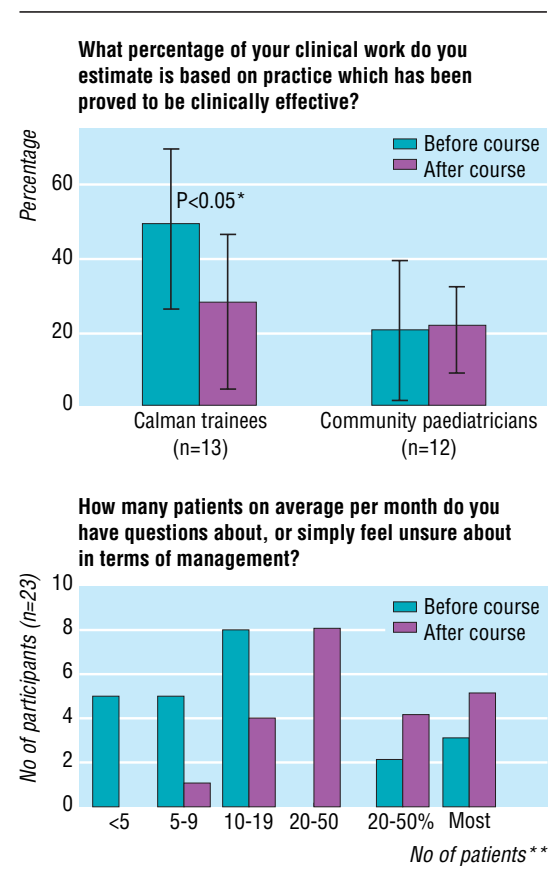

* Results shown as mean (SD), unpaired $t$ test used as questionnaires were anonymous

** Some participants answered as proportion of patients seen.

Questions asked of Calman trainees and community paediatricians before and after attending an evidence based child health course

estimate is based on practice which has been proved to be clinically effective?" and "How many patients on average per month do you have questions about, or simply feel unsure about in terms of management?" (figure).

Before the course participants reported that few questions arose during their clinical work, and the subgroup of Calman trainees were confident that a good proportion of their work was clinically effective. After the course the participants seemed to have developed a more questioning attitude in both paediatric practice and their management of patients' problems.

Clearly, a well planned study is needed to ascertain whether a course in evidence based medicine can really change the questioning behaviour of doctors in the long term. The data, however, do suggest that courses such as ours may make it acceptable to admit to insecurities about patient management and may foster an openness to asking questions.

One result of our course was that the community paediatricians, troubled by their lack of confidence in the effectiveness of their work, undertook and completed a study to ascertain the evidence base of community paediatrics similar to that carried out on a medical ward in Oxford. ${ }^{3}$

Barrie and Ward state that "evidence based medicine may make slow progress until doctors become more questioning in their routine practice." Our data provide some evidence (and hope) that simple investment in courses provided during medical training can change the prevailing 
culture and encourage doctors to take a more inquiring attitude towards the care and management of patients.

Mary C J Rudolf Consultant community

paediatrician

Department of Paediatrics and Child Health, University of Leeds, Leeds LS2 9NP

Karen Jones Research associate

Department of Primary Health Care, University of Newcastle, Newcastle upon Tyne NE1 7RU

1 Barrie AR, Ward AM. Questioning behaviour in general practice: a pragmatic study. BMJ 1997;315:1512-5. practice: a p

2 Sackett DL, Richardson WS, Rosenberg W, Haynes RB. Evidence based medicine: how to practise and teach EBM. Edinburgh: Churchill Livingstone, 1997.

3 Ellis J, Mulligan I, Rowe J, Sackett DL. Inpatient general medicine is evidence based. Lancet 1995;346:407-10.

\section{Time to publication of studies was not affected by whether results were positive}

Education and debate $\mathrm{p} 1519$

EDITOR-The time to publication measured by Stern and Simes began with approval of the project by the ethics committee; thus the interval embraced all phases of research and analysis. ${ }^{1}$ This interval differs from that used by several other ethics committees cited ${ }^{2}$ and in most previous reports of time to publication, which have begun with an analysis of completed data, not approval by the ethics committee. ${ }^{4}$

We examined the fate of all 493 completed research studies submitted by members of staff from 103 American medical schools for consideration to the 1991 meeting of the Society of Academic Emergency Medicine. ${ }^{5}$ We searched Index Medicus on line in 1996 to determine which studies had been published, and we sent questions to the authors of all unpublished studies. We evaluated the methodology and quality of all submitted abstracts using a blinded delphi panel, and we calculated the effect size ratio reported in each (percentage efficacy of the intervention divided by that of the control).

Altogether 179 studies of the 493 submitted were accepted for presentation; 223 of the submitted studies were published in 44 peer reviewed journals (39 in specialties other than emergency medicine), with impact factors of 0.23 to 24.5. The mean time to publication from the time of

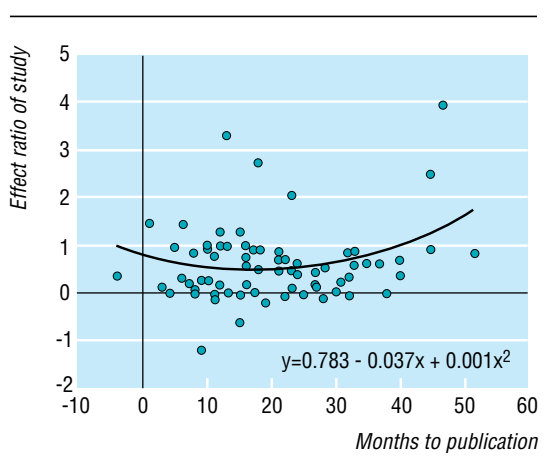

Relation of effect size ratio of studied treatment to speed of publication submission to the time of the meeting did not differ between controlled studies with positive outcomes and those with negative outcomes (19.1 (SD 12.5) months $v 15.6$ (11.7) months, $\mathrm{P}=0.20$ ). The impact factors of the publishing journals did not differ between studies with positive and negative results. Among published trials with control groups and a calculable effect size ratio, months to publication was poorly correlated with the effect size ratio $(r=0.13 ; r=-0.05$ for non-emergency medicine journals only) (figure). Stern and Simes obtained outcomes for $70 \%$ of eligible studies; we obtained them for all. The studies we examined had all completed the final analysis, whereas only $43 \%$ of the authors' eligible studies had. The largest difference, however, is probably that the publication interval in the authors' study incorporated all phases of the research, and its length may be due to researchers' enthusiasm for implementing logistics. Investigators finding early positive results may enrol patients faster, conclude studies and analyse results early, and submit the studies more promptly than other investigators. This represents a different phenomenon than that analysed previously and does not focus on bias by the journal or peer reviewer.

Michael L Callaham Professor of medicine Ellen Weber Associate professor of medicine Gary Young Associate professor of clinical medicine University of California, San Francisco, San Francisco, CA 94143-0208, USA

Robert Wears Associate professor of emergency medicine

University of Florida, Health Centre Jacksonville, 655 W 8th St, Jacksonville, FL 32209, USA

Chris Barton Associate professor of emergency medicine

University of North Carolina at Chapel Hill, CB

7594101 Manning Drive, Chapel Hill, NC

27599-7594, USA

1 Stern J, Simes R. Publication bias: evidence of delayed publication in a cohort study of clinical research projects. BMJ 1997;315:640-5. (13 September)

2 Easterbrook PJ, Berlin JA, Gopalan R, Matthews DR Publication bias in clinical research. Lancet 1991;337:867 72.

3 Dickersin K, Min YI, Meinert CL. Factors influencing publication of research results. Follow-up of applications submitted to two institutional review boards. JAMA 1992;267:374-8.

4 Yentis S, Campbell F, Lerman J. Publication of abstracts presented at anesthesia meetings. Can J Anaesth
1993;40:632-4.

5 Callaham M, Wears R, Weber E, Barton C, Young G. Positive-outcome bias and other limitations in the selection of research abstracts for a scientific meeting [abstract]. Acad Emerg Med 1997;4:480.

\section{$B M J$ has largely met its target for publication time for long papers \\ Education and debate $\mathrm{p} 1519$}

EDITOR-In an editorial in 1993 Smith declared the $B M / \mathrm{s}$ intention to become accountable to its readers by setting targets on editorial decision making and promising to publish the results of the audit. ${ }^{1}$ Audit results for the past four years were published last year. $^{2}$ To identify the factors that influence the time between acceptance and publication, I systematically reviewed the $B M J$ over four years.

I collected the following information for all papers published between January 1992
Country and city (for UK papers only) of affiliation of first authors, BMJ 1992-95 (leading 10)

\begin{tabular}{lccc} 
Country & No (\%) & City & No (\%) \\
\hline UK & $771(66.4)$ & - & - \\
\hline Australia & $46(4.0)$ & London & $223(29.1)$ \\
\hline Denmark & $40(3.4)$ & Oxford & $64(8.4)$ \\
\hline Netherlands & $38(3.3)$ & Glasgow & $38(5.0)$ \\
\hline USA & $33(2.8)$ & Birmingham & $29(3.8)$ \\
\hline Sweden & $33(2.8)$ & Bristol & $28(3.7)$ \\
\hline Finland & $30(2.6)$ & Aberdeen & $26(3.4)$ \\
\hline Italy & $26(2.2)$ & Cambridge & $26(3.4)$ \\
\hline France & $20(1.7)$ & Cardiff & $26(3.4)$ \\
\hline New Zealand & $12(1.0)$ & Leeds & $25(3.3)$ \\
\hline Norway & $11(0.9)$ & Edinburgh & $24(3.1)$ \\
\hline
\end{tabular}

and December 1995: publication date, date of acceptance of the paper, whether there was an accompanying editorial, other related papers in the same issue, and country and city (for papers from the United Kingdom (UK)) of affiliation of the first author. The data were analysed with SPSS version 6.1 .

A total of 1162 papers were published in 200 issues, giving an average of 5.81 per issue. Altogether 752 were full papers and 410 were short papers. Two thirds of the papers were from the UK (771) followed by Australia (46), Denmark (40), and the Netherlands (38). The table shows the 11 most common countries and 10 most common cities of affiliation of the first author of each paper. In a multiple linear regression model with $\log$ days to publication as a dependent variable, the following covariates were included: accompanying editorial (yes/ no), length of paper (short/long), other related papers (yes/no), and country of affiliation of first author (UK/non-UK). Publication time was relatively longer for short papers (median days for short and long papers 90 and 72, respectively; relative publication time $127 \%, \mathrm{P}<0.0001$ ) and relatively shorter for papers from the UK (median for UK and non-UK papers 74 and 78 days; relative publication time 93\%, $\mathrm{P}<0.003)$. No other variables reached significance. The median publication time for long papers was $68,71,79$, and 71 days for the four years studied. Three papers, all from the UK, were published more than a year after acceptance.

The $B M J$ has largely met its target for publication time for the long papers, though the short papers tend to take longer. As there are many medical schools and a large number of research organisations in London, nearly a third of UK publications are from London. The time from submission to publication could not be analysed as the $B M J$ does not publish the date of receipt of papers. I recommend publication of this date to aid future audit of the publication process.

I thank Dr T Prevost for statistical help. P Badrinath Specialist registrar in public health medicine

Walsall Health Authority, Walsall WS1 1TE

1 Smith R. Auditing BMJ decision making. BMJ 1993; 306:3-4.

2 BMJ audit: time to decision and publication. BMJ 1997; 314:1728. 